\title{
Lupus-like reactions in patients with inflammatory bowel disease: a little bit different data from another center
}

Daniella Pigniczki, Mariann Rutka, Klaudia Farkas*, Tamas Molnar*

* contributed equally

First Department of Medicine, University of Szeged Faculty of Medicine

Running head: Lupus-like reactions in inflammatory bowel disease

To the Editor,

We read the recently published article by Macaluso et al. examining the appearance of lupus-like reactions (LLR) in patients with inflammatory bowel disease (IBD), who were treated with anti-TNF drugs, with great interest (1). It is well-known, that IBD can be associated with extraintestinal manifestations, and that sometimes anti-TNF therapy can also generate paradox reactions, such as psoriasiform cutaneous reactions and arthritis (2), however LLR as a paradoxical reaction is a relatively new kid on the block. Our aim is to contribute to the knowledge of the rare-occuring LLRs with our experience.

The total of 183 IBD patients received infliximab (IFX) or adalimumab (ADA) between 2012 and the August of 2019 in our center, totally contributed 1403 person-years follow-up. During this interval, 3 patients [1.64\%; 2 women, 1 man; mean age at the diagnosis: 35.3 years (range: 26-51)] were presented with biological-therapy-induced-LLR according to the criteria of Macaluso et al, therefore the incidence rate of LRR was 2.14 per 1000 person-years. Female gender represents $66.7 \%$ of the LLR patients and $53.3 \%$ of the non-LLR group, while smokers represent $33.3 \%$ of the LLR patients and $12.3 \%$ of the non-LLR group. In regard to the low number of LLR patients, the value of these data has to be considered.

Two patients have Crohn's disease (CD) and one has ulcerative colitis (UC). All patients had IFX as the offending drug, which was the first-line biological treatment in two cases, while the remaining one patient had previously received ADA. All of them were in clinical remission at the time of the LLR diagnosis, but have experienced the most extended forms of IBD before: our UC paitent has already had pancolitis and our two CD patients have experienced ileocolic localisation with the involvement of the upper gastrointestinal tract as well. Arthralgia was reported in all 3 cases, while fatigue occurred in 2 cases, and cutaneous manifestation in 1 patient. None of the patients developed fever or serositis, but two of them experienced the symptoms of myositis showing muscular involvement, which was also confirmed by the 
elevated level of the muscle-specific Jo-1 autoimmune antibody in one case. Both ANA and anti-ds-DNA titers were elevated in 2-2 patients, respectively. Furthermore, other less common lupus-antibody titers also showed elevation: rheumatoid factor (2 patients), antiribosomal Pprotein antibody (1 patient), anti-nucleosome antibody (1 patient), and the drug-induced autoimmunity marker histone antibody (1 patient) were found. We have drug monitoring data from two patients. Both were presented with subtherapeutic IFX levels $(2.75 \mathrm{ng} / \mathrm{l}$ and $0.12 \mathrm{ng} / \mathrm{l})$ and anti-drug antibody positivity. Six months after the LLR diagnosis and consequent drug disposal, the control autoimmune titers remained higher than normal in all cases. All three patients received combination therapy with azathioprine. None of them received steroids when the diagnosis of LLR was set up, but all of them had LRR just after steroid withdrawal.

Our results can confirm the observation of Macalusco et al that LLR can exist more frequently among women and former smokers, however we hypothesize the role of drug antibody formation and steroid withdrawal in the development of LLR. Serum drug level measurement and an autoimmune panel should be performed after a one-year therapy, and in case of subtherapeutic drug level and elevated antibodies, a biological drug switch should be considered.

\section{References:}

1. FS Macaluso, C Sapienza, M Ventimiglia et al. Lupus-like reactions in patients with inflammatory bowel disease treated with anti-TNFs are insidious adverse events: data from a large single-center cohort. Scandinavian Journal of Gastroenterology. 2019. DOI: $10.1080 / 00365521.2019 .1663260$

2. L Puig. Paradoxical Reactions: Anti-Tumor Necrosis Factor Alpha Agents, Ustekinumab, Secukinumab, Ixekizumab, and Others. Curr Probl Dermatol. 2018;53:49-63. doi: 10.1159/000479475. 